\title{
Der erzählte Zaubertrank
}

\section{Eberhard Wolff}

Prof. Dr. rer. soc., Redaktor Kultur, Geschichte, Gesellschaft

Das Heldenepos ist bekanntlich eine in der populären Medizingeschichte endemische Gattung. Vor einhundert Jahren, zu Ende des Ersten Weltkriegs, zog die verheerende "Spanische Grippe» in drei Wellen (1918/19) über Europa und liess mehr Menschen sterben als ebendieser Krieg. Weltweit erlagen ihr bis zu 50 Millionen Menschen, davon etwa 25000 in der Schweiz. Das war keine gute Zeit für das Schreiben von Heldengeschichten. Eine eigentliche Therapie dieser Virusinfektion gab es damals ebenso wenig wie eine durchgreifende Prävention. Vor diesem Hintergrund

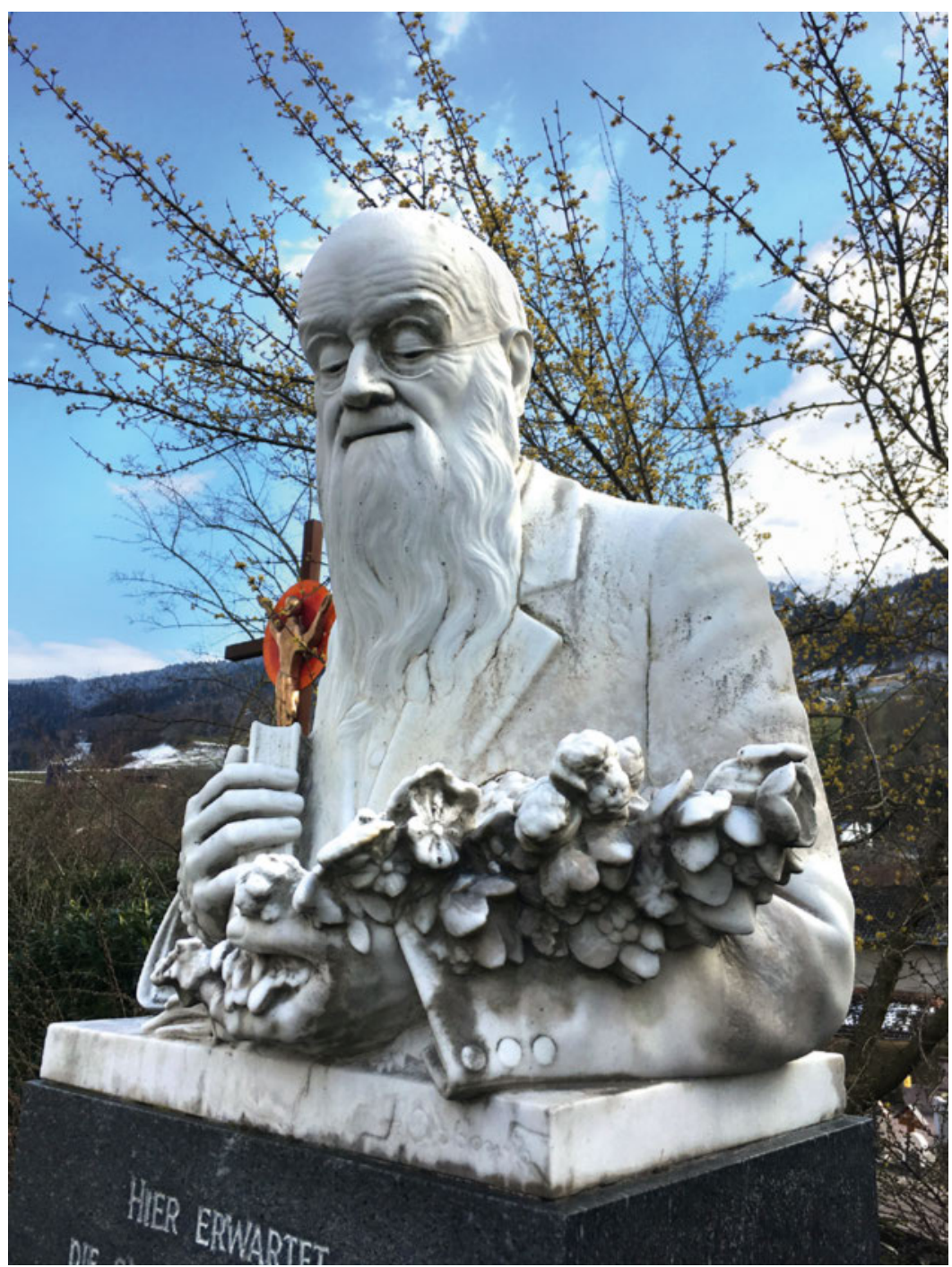

Die Überhöhung von Künzle begann bereits zu Lebzeiten und stieg nach seinem Tod: Grabmal von Johann Künzle auf dem Friedhof von Wangs SG. probierten Ärzte eher hektisch und unsystematisch verschiedenste alte und neue Therapieformen aus und machten letztlich keine gute Falle [1,2]. Umso mehr versuchten die damals populären Naturheiler, die Nützlichkeit ihrer Verfahren aufzuzeigen, um so auch Anerkennung und vielleicht ein Stück Heldentum zu ergattern.

\section{Johann Künzle, Priester und Heiler}

Im Jahr 1918 war der «Kräuterpfarrer» Johann Künzle (1857-1945) aus dem sankt-gallischen Wangs bereits eine weithin prominente Persönlichkeit. Sein Bestseller «Chrut und Uchrut», ein "praktisches Heilkräuterbüchlein» von 1911, hatte sich schon mehrere hunderttausend Mal verkauft. Der deutsche Kulturkritiker und Philosoph Walter Benjamin nahm dieses Heft 1931 dann sogar als das Beispiel für seine Überlegungen, weshalb eine Publikation ein grosser Erfolg werden könne. Inhaltlich allerdings verglich er es ironisch mit «Kraut und Rüben».

Neben seinem Priesteramt betätigte sich Künzle als Heiler: Viele Menschen glaubten an seine Ideen und strömten zu ihm. Im Ort Wangs hatte er ein Zentrum für das Sammeln und den Vertrieb von Kräutern aufgebaut.

\section{Der Kräutertee}

Zur aufkommenden Grippe-Epidemie entwickelte Künzle dann einen speziellen Grippetee mit Stechpalmenblättern, Wiesensalbei und Wermut [3], den er an die umliegende Bevölkerung verkaufte oder verteilte. Die Grippe selbst glaubte Künzle schon Tage vor dem Erscheinen ihrer ersten Symptome diagnostizieren zu können: mit der Technik des Pendelns - wofür er seine goldene Taschenuhr verwendete.

Den Kristallisationskern für die Heldengeschichte gab dann ein zeitgenössischer Bericht, demzufolge damals in Wangs niemand an der Grippe gestorben sei. So genau kann man das jedoch nicht sagen. Der Historiker und Künzle-Biograph Beat Frei [4] ist der Geschichte nachgegangen und hat im Totenbuch eine Barbara Grünenfelder als Grippetote entdeckt. Bereits während der Epidemie wurde um diese Frage öffentlich gestritten. War der Gemeinderatsschreiber Jakob Kalberer 


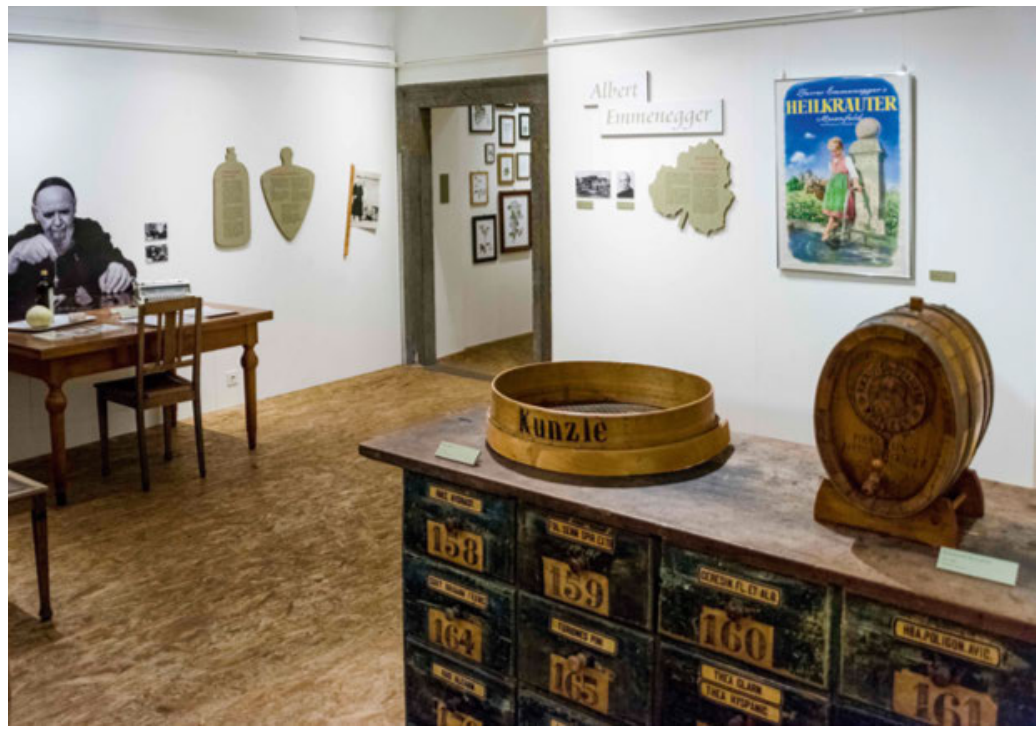

\section{Ausstellung über Kräuterdoktoren und Kabispater}

Eine sehenswerte Sonderausstellung über das Heilen mit Pflanzen zeigt das Rätische Museum bis zum Herbst in Chur. Im Mittelpunkt steht das spezielle Phänomen der Kräuter-Geistlichen in Graubünden. Der Abstimmungskampf um Künzles Heiltätigkeit wird darin genauso eindrucksvoll gezeigt wie die industrielle Vermarktung seiner Produkte. Der "Kabispater» Thomas Häberle (1912-1997) verschrieb in den Klöstern von Müstair und Disentis Kohl und Olivenöl, und als "Pendelpater» wollte er Krankheiten und Wasseradern auf die Spur kommen. Heute ist der Umgang mit Kräutern weniger exotisch. Sie sollen der Gesundheit, der Schönheit, dem Genuss und damit letztlich auch dem Tourismus dienen.

«Kräuterdoktor - Kabispater. Heilen mit Pflanzen in Graubünden». Bis 9. September 2018 im Rätischen Museum Chur, Di bis So 10-17 Uhr, www.rm.gr.ch

damals der Grippe erlegen, wie es der freisinnige politische Kontrahent von Künzle, Leonz Schumacher, in der Zeitung vermutet hatte? Oder war es etwas anderes, wie Künzle meinte und eine politische Attacke von Schumacher vermutete? Im Totenbuch steht «Nierenwassersucht» als Todesursache, aber das hatte Künzle selber in seiner Funktion als Priester eingetragen.

\section{Einem zeitgenössischen Bericht zufolge sei} damals in Wangs niemand an der Grippe gestorben - so genau kann man das jedoch nicht sagen.

Detailliert nachzulesen ist dies - in literarisch aufbereiteter Form - seit dem letzten Jahr auch im biographischen Künzle-Roman «Uns Menschen in den Weg gestreut» der Autorin Marianne Künzle, die nicht mit dem Kräuterpfarrer verwandt ist [5].

\section{Künzles Tee und der Grippetod}

Und selbst wenn in Wangs alle Grippekranken überlebt haben, bleibt die Frage, ob sie tatsächlich wegen
Künzles Tee mit dem Leben davongekommen sind. Wissen wir, ob die von Künzle versorgten Wangser den Tee auch wirklich genommen haben? Warum sind diejenigen, die ihn nicht genommen haben, offenbar auch nicht gestorben? Damals wurde eben keine kontrollierte Arzneimittelstudie durchgeführt. Und selbst eine seriöse Studie hätte nur Koinzidenzen, aber keine Kausalitäten festhalten können. Wie sollte man wissen, dass es gerade der Kräutertee war, der vor dem Grippetod schützte? Wie viele von denen, die ihn genommen hatten, wären ohne ihn gestorben? Schon damals liess sich nicht definitiv nachweisen, dass wegen Künzles Grippetee in Wangs (möglicherweise) niemand an der Grippe gestorben ist. Und heute noch viel weniger. Für die damaligen Therapien der "Schulmedizin» gilt übrigens weitgehend das Gleiche [1].

Die ganze Sache wäre wohl wieder in Vergessenheit geraten, wäre sie nicht so sehr von damals brennenden Streitfragen befeuert gewesen, die bis heute nachwirken: Ist die Kräuterheilkunde nützlich? Hat die Schulmedizin in der Spanischen Grippe ihr Versagen bewiesen? Sollen Nichtärzte, ja Priester, therapieren dürfen? Kann man einen Priester, der "pendelt», noch ernstnehmen? Schliesslich heizten die letzten Ausläufer des «Kulturkampfs» den Streit an: Sollten nun die Katholiken oder die Freisinnigen mehr politisches Sagen vor Ort haben?

\section{Eine Geschichte - drei Varianten}

Die Geschichte mit den ausgebliebenen Wangser Grippetoten und Künzles Grippetee wurde und wird bis heute überliefert. Sie wird aber auf ganz unterschiedliche Art «erzählt». Da gibt es die skeptisch-vorsichtige Variante der distanzierten Chronisten. Die Ortschronik von 1985 vermeldet zum Beispiel, dass damals niemand an der Grippe gestorben sein «soll» [6]. Auch die historisch angelegten Biographien [8], Marianne Künzles detailliert recherchierter Roman und eine ganz aktuelle Ausstellung in Chur (siehe Infokasten) lassen mit Bedacht viele Fragen offen. Glühende Anhänger erzählen dagegen eine unzweideutige Heldengeschichte. Zum 100. Geburtstag des Kräuterpfarrers schrieb die Künzle-Nichte Christine Abbondio im urchigen Dialekt: «Anno 1918, won e schrecklichi GrippeEpidemi pestartig au i sim Sprengel gwüetet hät, ist Dank em Pendel und de Awendige vo sim spezielle Grippetee vo vierhondert Patiente i siner Pfarrei kein einzege gstorbe» [7]. Der Pfarrer hatte seiner Nichte übrigens schon zu Lebzeiten seinen Kräuterhandel übertragen. Im Gegenzug lenkte sie das Andenken an ihren Onkel in «mythische Bahnen» [4]. 


\section{Mythos und Wirklichkeit}

Mit ihrer Weitergabe heizte sich die Heldengeschichte manchmal noch auf. Im Werbetext des Zytglogge-Verlags wird aus Marianne Künzles detailliert-vorsichtiger Nacherzählung ein knallig-eindeutiges: «Nur in der sanktgallischen Pfarrgemeinde Wangs starb niemand, denn hier tranken alle eine von Künzle angefertigte Teemischung.» Damit nicht genug. Im Übergang vom Werbetext zu den Presseartikeln wird eine «legendäre» Erfolgsgeschichte daraus, eine "Sensation» [9], bei der Künzle die Wangser nicht nur vor Grippetoten, sondern gleich vor der ganzen Grippe geschützt hat. Der Kräuterpfarrer mutiert zum «Kräuterpapst» [10]. Am Ende kann ein Artikel mit der Erfolgsstory sogar Werbung für Alpenkräuter-Likör machen [11].

Einige der durch Zeitungen und Internet geisternden Künzle-Heldengeschichten enden damit, dass die Wangser ihren Kräuterpfarrer als Dank für ihre Rettung im Jahr 1918 zum Ehrenbürger gemacht hätten

\section{Mit ihrer Weitergabe heizte sich die Helden- geschichte manchmal noch auf.}

[10]. Das tönt einleuchtend. Das Problem dabei ist nur, dass es offenbar nirgends einen Nachweis dafür gibt. Weder die Ortsgemeinde noch der Gemeinderatsschreiber konnten auf Nachfrage in den alten Akten etwas finden [12]. Immerhin erhielt Künzle das Wangser Ortsbürgerrecht. Das mag eine Ehrenbezeugung gewesen sein, aber war leider schon 1912, sechs Jahre vor Grippe und Stechpalmentee.

\section{Wangs - ein "gallisches Dorf»?}

Ich werde den Verdacht nicht los, dass es der Wunsch nach einer Heldengeschichte war, der an der Narration "weitergeschrieben» hat. Die aber könnte zumindest ein ergreifendes Drehbuch abgeben. Es könnte beginnen mit einer Stimme aus dem "Off»: «Wir befinden uns im Jahre 1918 n.Chr. Ganz Europa ist von der Spanischen Grippe besetzt ... Ganz Europa? Nein! Ein von unbeugsamen Sarganserländern bevölkertes Dorf hört nicht auf, dem Eindringling Widerstand zu leisten.» Dann Auftritt des Kräuterhelden (eine Mischung aus Asterix und Druide) mit seinem Zaubertrank usw. usw. Das könnte als sommerliches Freiluft-Musical im Heidiland gut funktionieren - mit einer Flasche Kräuterlikör im VIP-Package.

Aber wer spielt dann die Römer, die verhauen werden? Die kommen erst im zweiten Teil der Geschichte vor, gespielt von der Bündner Regierung und Ärzteschaft des Jahres 1922, als sie eine Abstimmung um das Recht Künzles zu praktizieren verloren.

\section{Literatur}

1 Lauterburg B. Die schlimmste Überraschung war die absolute Ohnmacht ... Schweiz Ärzteztg.2018;99(21):681-3.

2 Bauer F, Vögele J. Die «Spanische Grippe» in der deutschen Armee 1918: Perspektive der Ärzte und Generäle, in: Medizinhistorisches Journal 48, Heft 2 (2013), 117-52, S. 137. Siehe auch die Arbeiten von Wilfried Witte zur Grippe-Geschichte.

3 Ich danke dem Präsidenten des Kräuter-Pfarrer Künzle Vereins, Herrn Louis Hüppi, Vilters, für diese Information.

4 Frei B. Wangs und sein Kräuterpfarrer. Wangs: Katholische Kirchengemeinde, 2007.

5 Künzle M. Uns Menschen in den Weg gestreut. Kräuterpfarrer Johann Künzle (1857-1945). Bern: Zytglogge 2017. 351 S., CHF 36.

6 Vogler W. Wangs: Dorf, Nachbarschaft, Gemeinde. Wangs: Ortsgemeinde 1985.

7 Abbondio-Künzle C, Schmidt GO. Gedenkschrift zum 100. Geburtstage des Chrüterpfarrers Johann Künzle 1857-1945. Fryburg (Freiburg): Schwyzerlyt-Verlag 1957, 19.

8 Fliri M. Kräuterpfarrer Johann Künzle - zwischen Publizistik und Kräuterheilkunde. In: Archiv der Diözese Feldkirch (Hg.): Chrut und Uchrut vergönt ned. Biografien in der Schnittmenge Publizistik, Kräuterheilkunde und Kulturkampf zur Jahrhundertwende in Feldkirch und darüber hinaus. Feldkirch: Eigenverlag 2015, S. 56-73.

9 https://spuren.ch/content/magazin/single-ansicht-nachrichten/ datum////naturkraft-schweiz-die-guten-tipps.html.

10 Zimmermann ML. Das bewegte Leben des Kräuterpapstes. Berner Zeitung, 27. Juni 2017. https://www.bernerzeitung.ch/kultur/buecher/Das-bewegte-Leben-des-Kraeuterpapstes/story/21113493.

11 Ganzfried D. Ein Schluck Heimat. Schweizer Familie 7/2016; 40-4. http://www.ganzfried.ch/PDF/leseproben/Glarner_Alpenbitter. pdf.

12 Mein herzlicher Dank geht an Frau Regula Gugg, Ratsschreiberin der Ortsgemeinde Wangs, und Herrn Patrik Schlegel, Gemeinderatsschreiber der Gemeinde Vilters-Wangs, für die aufwendige Recherche.

\section{Bildnachweis}

Foto Grabmahl: @ Eberhard Wolff

Foto zur Ausstellung: zVg vom Rätischen Museum Chur 